\section{Patienten mit Organversagen: Versorgung am Lebensende verbesserungsbedürftig}

Ein Lebensende ohne intensivmedizinische Maßnahmen zusammen mit einer hochwertigen Palliation kommt bisher vor allem Krebs- und Demenzpatienten zugute. Um Patienten mit Organversagen kümmert sich die Intensivmedizin vielleicht immer noch zu lange.

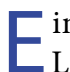
ine qualitativ hochwertige Pflege am Lebensende ist nicht nur für Krebspatienten wichtig. Auch die letzten Lebenswochen von Patienten mit anderen schweren Erkrankungen erfordern eine hochwertige palliativ-pflegerische Begleitung. Ein Messinstrument für die Qualität der End-of-Life-Betreuung ist die Einschätzung durch Familienangehörige. Dieses Instrument setzte jetzt eine retrospektive US-amerikanische Querschnittsstudie ein, in der nach positiv empfundenen Charakteristika von Maßnahmen am Lebensende bei verschiedenen Grunderkrankungen gesucht wurde.

Einbezogen in die Auswertung waren insgesamt 57.753 sterbende Patienten unter anderem mit den Diagnosen Nierenerkrankungen im Endstadium,
Krebs, Herz-Lungen-Versagen, Demenz und Altersschwäche sowie 34.005 Familien dieser Patienten. Ergebnis: Von den Patienten mit Nieren-, Herz- und Lungenerkrankungen erhielt rund die Hälfte eine palliative Betreuung im Vergleich zu $73 \%$ der Krebspatienten und $61 \%$ der Demenzpatienten $(\mathrm{p}<0,001)$. Rund ein Drittel der Patienten mit Nieren-, Herzund Lungenerkrankungen starb auf der Intensivstation (Nierenversagen 32,3\%, kardiopulmonales Versagen 34,1\% und Altersschwäche 35,2\%) im Vergleich zu rund $10 \%$ der Patienten mit Krebs oder Demenz (13,4\% bzw. 8,9\%; p<0,001). Dass die Qualität der Pflege am Lebensende exzellent gewesen sei, bescheinigten 59,2\% beziehungsweise 59,3\% der Familienangehörigen von Krebs- beziehungsweise Demenzpatienten, aber sig- nifikant weniger Familienangehörige von Patienten mit anderen Erkrankungen (Nierenerkrankungen 54,8\%, kardiopulmonales Versagen $54,8 \%$ und Altersschwäche 53,7\%; alle $\mathrm{p}<0,02$ vs. Krebspatienten). Der Eindruck einer qualitativ besseren Pflege bei den Krebsund Demenzpatienten beruhte vor allem auf einer palliativmedizinischen Betreuung, dem Setting während des Sterbens und der Ablehnung von wiederbelebenden Maßnahmen.

Fazit: Die von Familienangehörigen beurteilte Pflegequalität am Lebensende war für sterbende Patienten mit Krebs oder Demenz signifikant besser als für Patienten mit Nieren-, Herz- oder Lungenerkrankungen. Einfluss auf die Einschätzung hatten vor allem die höheren Raten von palliativen Pflegeeinsätzen, die Verweigerung von wiederbelebenden Maßnahmen und ein Sterben außerhalb von Intensivstationen.

Dr. Barbara Kreutzkamp

Wachteman MW et al. Quality of end-of-life care provided to patients with different serious illnesses. JAMA Intern Med 2016; 176:1095-1102

\section{Opioidverordnungen nach schmerzhaften Operationen langfristig erhöht}

\author{
Lassen sich Patienten nach operativen Eingriffen tatsächlich häufiger Opioide \\ verschreiben als Patienten ohne einen schmerzhaften Eingriff? Und wenn ja, \\ was sind die Risikofaktoren für einen postoperativen Opioidmissbrauch? Eine \\ US-amerikanische Registerstudie gibt Antworten.
}

V or allem in den USA belastet ein zunehmender Opioideinsatz die Gesundheit der Patienten und das Gesundheitswesen. Als ein Risikofaktor für diesen Trend gelten Operationen mit perioperativer Opiodgabe. Um die RisikoDatenlage speziell bei opioidnaiven Patienten zu verbessern, durchforsteten US-Forscher die Krankenakten von opioidnaiven Operationspatienten hinsichtlich Folgeverordnungen von Opioiden im ersten Jahr nach dem Eingriff.

Einbezogen in die Registerstudie waren 641.941 privatversicherte Patienten im Alter von 18 bis 64 Jahren, bei denen schmerzhafte Eingriffe vorgenommen wurden. Dazu gehörten arthroplastische Operationen, Cholecyst- und Appendektomien, Kaiserschnitte, Mastektomien und transurethrale Prostataresektionen. Ausgewertet wurden die postoperativen Opioidverordnungsdaten von präoperativ opioidnaiven Patienten, bereinigt um mögliche konfundierende Faktoren wie Geschlecht, Alter, psychiatrische Diagnosen, Alkoholabusus und Benzodiazepineinnahme. Die so ermittelten Patienten wurden einer Kontroll- gruppe von 18.011.137 nicht operierten Patienten gegenübergestellt.

Ergebnis: Die meisten der untersuchten schmerzhaften operativen Eingriffe erhöhten das Risiko für einen gesteigerten postoperativen chronischen Opioidgebrauch mit Odds ratios von 1,28 (95\%-KI 1,12-1,46) bei einem Kaiserschnitt bis zu 5,10 (95\%-KI 4,67-5,58) bei einer Knie-Arthroplastie. So rangierte die Inzidenz von chronischem Opioidgebrauch, definiert als mehr als zehn Opioidverordnungen über mehr als 120 Tage, bei den opioidnaiven operierten Patienten zwischen 0,119\% bei Frauen nach einem Kaiserschnitt (95\%-Konfidenzintervall 0,104-0,134\%) bis $1,41 \%$ nach einer Knie-Arthroplastie (95\%-KI 1,29-1,53\%). Lediglich bei Kataraktoperationen, laparoskopischer Appendektomie, endoskopischer SinusOperation und transurethraler Prostataresektion waren keine erhöhten Inzidenzen für einen ostoperativen Opioid- 